Methods: Twelve TNFi naive AS patients (female 7/12; age 39 \pm 11 years) with high disease activity (BASDAI $5.5 \pm 1.1$ ) were included. $\left[{ }^{18} \mathrm{~F}\right]$ fluoride PET-CT scans were performed before initiation of TNFi therapy. In 2 patients, biopsies were obtained from PET identified spine lesions for histologic analysis. Of the remaining 10 patients, a second $\left[{ }^{18} \mathrm{~F}\right]$ fluoride PET-CT scan was performed after 12 weeks of TNFi treatment. PET scans were scored visually for positivity by two blinded expert readers. Subsequently, $\left[{ }^{18} \mathrm{~F}\right]$ fluoride uptake was quantified in PET positive $\left(\mathrm{PET}^{+}\right)$lesions using the standardized uptake value corrected for individual integrated whole blood activity concentrations $\left(S_{U} U V_{A U C}\right)$. Clinical response to TNFi was defined according to ASAS20 at 24 weeks.

Results: At baseline, in all patients at least one axial $\mathrm{PET}^{+}$lesion was found. In spine, 6/10 patients showed 84 lesions (range 2-30;63\% thoracic spine, primarily costovertebral joints) and in the $\mathrm{SI}$ joints in $9 / 10$ patients were $\mathrm{PET}^{+}$ (Fig A). Histological analysis of PET+ lesions in the spine confirmed local osteoid formation, which was nearly absent in PET negative lesions. Quantitative PET analysis revealed significantly lower $\left[{ }^{18} \mathrm{~F}\right]$ fluoride uptake in spine lesions at baseline in responders than in non-responders. This difference remained after 12 weeks of treatment (mean difference in SUV Auc : $-0.5,95 \% \mathrm{Cl}:[-$ $0.7,-0.2], p=0.001)$. After 12 weeks of TNFi treatment, $\left[{ }^{18} \mathrm{~F}\right]$ fluoride uptake in clinical responders decreased significantly in the costovertebral (mean difference

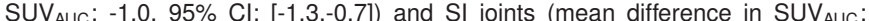
$-1.2,95 \% \mathrm{Cl}:[-2.3,-0.2]$ ) (fig B) in contrast to non-responders (mean difference in SUV ${ }_{\text {AUC }}:-0.4,95 \% \mathrm{Cl}:[-2.3,1.6]$ and $+0.4,95 \% \mathrm{Cl}$ : $[-0.6,1.4]$, respectively). $\left[{ }^{18} \mathrm{~F}\right]$ fluoride uptake in other spinal lesions such as bridging syndesmophytes showed heterogeneous response without a significant decrease in $\left[{ }^{18} \mathrm{~F}\right]$ fluoride accumulation over time at a group level.

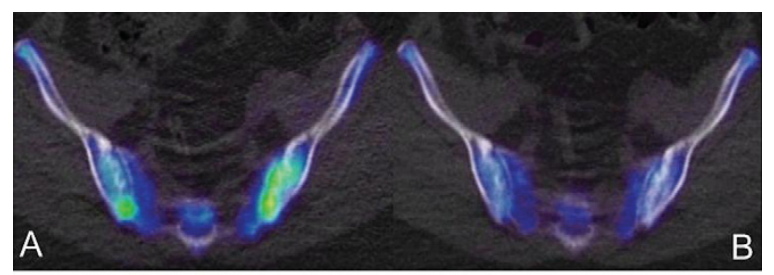

Figure: Example of $\left[{ }^{18} \mathrm{~F}\right]$ fluoride uptake in both $\mathrm{SI}$ joints at baseline $(\mathrm{A})$ and a clear decrease of uptake after 12 weeks of TNF-I (B).

Conclusions: $\left[{ }^{18} \mathrm{~F}\right]$ fluoride PET-CT enables non-invasive visualization of (changes in) lesions with bone formation of the whole spine and SI joints in clinically active AS patients, which is confirmed by histological signs of osteoid formation. Part of these lesions, in particular costovertebral lesions in spine and SI joints, decreased in clinical responders to TNFi (and not in non-responders), whereas other spinal lesions remained unchanged at a group level.

Disclosure of Interest: None declared

DOI: 10.1136/annrheumdis-2017-eular.2004

\section{FRI0624 STRUCTURAL MRI-BASED CONNECTOMICS IN SLE: A PILOT STUDY}

G.A. Ramirez ${ }^{1,2}$, E.P. Bozzolo ${ }^{1}$, P. Preziosa ${ }^{3}$, M.A. Rocca ${ }^{3}$, L. Moiola ${ }^{3}$ L.A. Coletto ${ }^{2}$, E. Tombetti ${ }^{1,2}$, P. Rovere-Querini ${ }^{1,2}$, M. Filippi ${ }^{2,3}$

A.A. Manfredi ${ }^{1,2}{ }^{1}{ }^{1}$ Unit of Allergy, Immunology, Rheumatology and Rare

Diseases, IRCCS Ospedale San Raffaele; ${ }^{2}$ Università Vita-Salute San Raffaele ${ }^{3}$ Institute of Experimental Neurology and Unit of Neurology, IRCCS Ospedale San Raffaele, Milano, Italy

Background: Neuropsychiatric manifestations are common in patients with systemic lupus erythematosus (SLE). Furthermore, subclinical brain damage occurs in an even higher fraction of patients. However, little is known about the effect of these phenomena on brain connectivity. MRI-based connectomics relies on graph analysis of structural and functional images to detect alterations of the topographic organization of the brain and has been successfully employed to dissect network disassembly in neuroinflammatory diseases such as multiple sclerosis (MS) and Devic's syndrome.

Objectives: To investigate the topographic organization of the brain of patients with SLE with and without neuropsychiatric manifestations.

Methods: Thirty-two patients with SLE (12 with overt neuropsychiatric involvement as per ACR criteria) were enrolled and compared with 32 healthy controls (HC) and 32 patients with relapsing-remitting MS, all matched for sex, age and disease duration (where applicable). Diffusion tensor (DT) and dual-echo MRI scans were obtained. Structural connectivity matrices between 116 cortical and subcortical brain regions were estimated and global and nodal network metrics were calculated.

Results: Conventional MRI revealed that patients with SLE had significantly higher T2-lesional volumes when compared to controls $(p<0.0001)$. Patients with definite NPSLE had a higher lesion burden $(\mathrm{p}=0.006)$. Network strength, transitivity and global efficiency were all significantly impaired in patients with MS and SLE when compared to HC $(p<0.0001)$. MS and SLE were also characterized by higher average path length when compared to $\mathrm{HC}(p<0.0001)$. Global structural alterations were more significant in MS patients than in patients with SLE ( $p$ from 0.005 to 0.01 at multiple comparison). However, antiDNA-positive patients $(n=24)$ showed a more severe phenotype when compared to antiDNA-negative patients ( $p$ from 0.026 to 0.041 ) and did not differ significantly from patients with MS. When regional hubs were analysed, patients with SLE and MS showed a reduced strength compared to $\mathrm{HC}$ ( $\mathrm{p}$ from $<0.0001$ to 0.001 at multiple comparison). Hub strength impairment was more pronounced in MS when compared to SLE and preferentially involved hubs located in fronto-temporo-parieto-occipital cortices, subcortical nuclei (including the thalamus, caudate nucleus and putamen) and cerebellum ( $\mathrm{p}$ from 0.001 to 0.05 at multiple comparison). No significant associations were found between global structural parameters, clinical diagnosis of neuropsychiatric SLE, other SLE sub-phenotypes, presence of antiphospholipid antibodies, antiphospholipid syndrome and SLE-related damage burden.

Conclusions: Structural alterations of global and regional brain connectivity occur in patients with SLE, irrespectively of the clinical phenotype. AntiDNA-positive patients are characterized by a more severe phenotype, which is similar to that of patients with relapsing-remitting MS.

References:

[1] Sabbadini MG et al, Lupus, 1999.

[2] Filippi M et al., Lancet Neurol, 2013.

[3] Rocca MA et al., Brain Struct Funct, 2016.

Disclosure of Interest: None declared

DOI: 10.1136/annrheumdis-2017-eular.4264

\section{FRI0625 IMPROVEMENT OF JOINT INFLAMMATION AS ASSESSED BY MRI AND POWER DOPPLER ULTRASOUND (PDUS) IN AN OPEN LABEL STUDY IN PATIENTS WITH ACTIVE PSORIATIC ARTHRITIS TREATED WITH SECUKINUMAB (PSARTROS)}

E. Kampylafka, I. D'Oliveira, C. Linz, V. Lerchen, M. Englbrecht, A. Kleyer, J. Rech, G. Schett, A.J. Hueber. Department of Internal Medicine 3 Rheumatology and Immunology, Friedrich-Alexander-University

Erlangen-Nürnberg (FAU) and Universitätsklinikum Erlangen, Ulmenweg 18 , 91054 Erlangen, Germany, Erlangen, Germany

Background: Secukinumab, an anti-interleukin 17A monoclonal antibody, showed significant improvement of signs and symptoms of psoriatic arthritis (PsA) in FUTURE 1 study. Available studies used conventional radiography, not allowing a deeper imaging analysis of the inflammatory changes during application.

Objectives: To assess short term efficacy of secukinumab on inflammation and structural damage according to change in OMERACT-EULAR ultrasound score and the MRI PsAMRIS score in PsA patients.

Methods: PsA patients with active disease (TJC and SJC $>3$ ), were included in the 24 week open label prospective PSARTROS study and treated with subcutaneous secukinumab $300 \mathrm{mg}$ once weekly over 4 weeks, then once every 4 weeks. Baseline 1,5T MRI hand scans and ultrasound imaging of 28 joints were performed at baseline and after 24 weeks of treatment. MRI was scored according to PsAMRIS, ultrasound for synovial hypertrophy and Doppler activity according to OMERACT scores. Statistical significance was set at $\mathrm{p}<0.05$.

Results: 20 patients, mean age $52 \pm 9.9$ years, $60 \%$ female, mean disease duration $6.7 \pm 5.9$ years, $50 \%$ naïve for biological therapy, were included in the study. Three patients were early discontinued (recurrent pharyngitis, lack of efficacy, withdrawal of consent), and were not included into the longitudinal analysis. Baseline DAS28 was $5.03 \pm 0.96$, baseline DAPSA was $32.2 \pm 12$. 1 . On baseline MRI, all patients had at least one inflammatory sign (synovitis: $90 \%$, osteitis: $20 \%$, periarticular inflammation: $25 \%$, flexor tenosynovitis: $35 \%$, bone proliferation: $30 \%$, erosions: $60 \%$ ). Baseline composite PsAMRIS score

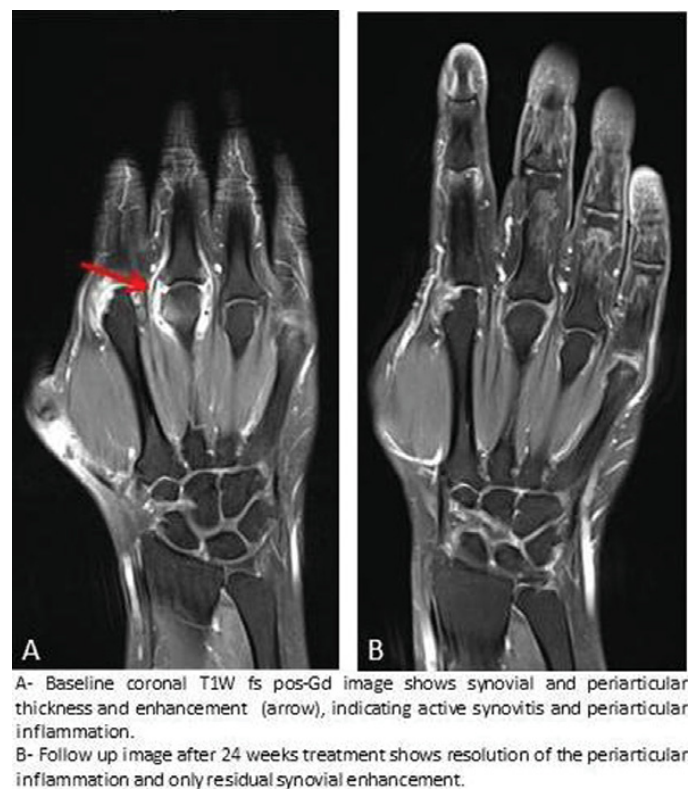


was 11.6 \pm 12.8 and baseline PsAMRIS synovitis score was $3.7 \pm 3.3$. Baseline ultrasound synovial hypertrophy and Doppler activity were $6.2 \pm 4.5$ and $3.5 \pm 4.0$, respectively. Specific MRI and ultrasound scores were significantly correlated with DAS28 and DAPSA at baseline. Clinical disease activity parameters significantly improved at follow up (DAS28: 2.94 $\pm 0.95, p<0.001$; DAPSA: $8.8 \pm 5.8, p<0.001$ ). PsAMRIS synovitis score $(2.5 \pm 2.4)$ as well as composite PsAMRIS score $(8.8 \pm 10.0)$ decreased longitudinally with secukinumab treatment $(\mathrm{p}=0.034$ and $p=0.039$, respectively). There was no progression in erosion or proliferation scores between baseline and follow-up. Synovial hypertrophy and Doppler activity in ultrasound also significantly improved with secukinumab treatment $(2.3 \pm 3.5$; $\mathrm{p}=0.009$ and $1.8 \pm 2.7 ; \mathrm{p}=0.003$, respectively). A significant percentage of patients reaching minimal disease activity showed residual signs of synovitis in the MRI and US (66\% and $50 \%$, respectively).

Conclusions: Secukinumab significantly improves MRI and ultrasound signs of joint inflammation in patients with PsA.

Acknowledgements: This study was supported by an unrestricted grant from Novartis.

Disclosure of Interest: None declared

DOI: 10.1136/annrheumdis-2017-eular.5094

\section{FRI0626 ANALYSIS OF CORRELATION AND CAUSES FOR DISCREPANCY BETWEEN QUANTITATIVE AND SEMI-QUANTITATIVE DOPPLER SCORES IN SYNOVITIS IN RHEUMATOID ARTHRITIS}

H. Rezaei ${ }^{1}$, E. af Klint ${ }^{1}$, H.B. Hammer ${ }^{2}$, L. Terslev ${ }^{3}$, M.-A. D'Agostino ${ }^{4}$ Y. Kisten ${ }^{5}$, L. Arnaud ${ }^{5} .{ }^{1}$ Rheumatology department, Institute of Medicine, Karolinska University Hospital, Stockholm, Sweden: ${ }^{2}$ Rheumatology, Diakonhjemmet Hospital, Oslo, Norway; ${ }^{3}$ Center for Rheumatology and Spine Disease, Rigshospitalet, Copenhagen, Denmark; ${ }^{4}$ Rheumatology, APHP, Hôpital Ambroise Paré, Saint-Quentin en Yvelines, Paris, France; ${ }^{5}$ Unit of Clinical Therapy Research, Inflammatory Diseases (ClinTRID), Institute of Medicine, Karolinska Institutet, Stockholm, Sweden

Background: Doppler US is used for the evaluation of synovitis in RA. The amount of Doppler signals are measured in the synovial tissue according to either semi-quantitative (SQS) or quantitative scoring (QS) methods. None of the SQS has been chosen by consensus so far, and this creates some heterogeneity in US in clinical practice and research. Conversely, a major strength of the QS is to allow objective measurement of Doppler pixels using a continuous numeric scale.

Objectives: This study aimed to evaluate the association between $\mathrm{SQS}^{1,2}$, and $\mathrm{QS}^{3}$ in RA patients with active disease. Additionally, to elucidate the reasons for potential discrepancies between SQS and QS assessments, in order to better understand the intrinsic limitations of these methods,

Methods: Adult patients with RA and inadequate clinical response to antirheumatic therapy were examined with US. Dorsal US of the wrists, MCP and MTP 2-5 were performed. US images with sign of synovitis were collected and the QS was measured. Five assessors blinded to the QS evaluated the images independently, according to either SQS method. Association between QS and SQS was studied using correlations and multilevel models taking into account the clustering of ratings at the rater, patient and joint levels.

Based on the cut-offs, the discrepant cases were extracted, and each participant was asked to re-grade his/her own discrepant cases, blinded to the initial SQS grading and original QS, and to provide an explanation for the discrepancy. Then, discrepant images and explanations provided were reviewed in consensus and classified into a limited number of categories

Results: Analysis of the 1190 ratings revealed a strong correlation ( $\rho=0.89$, $p<0.0001)$ and significant associations $(p<0.0001)$ between QS and SQS. Correlations between QS and SQS according to Szkudlarek et al. ( $\rho=0.87$, $\mathrm{p}<0.0001)$ or Hammer et al. $(\rho=0.91, \mathrm{p}<0.0001)$ were similar. A total of 239 $(20.1 \%)$ images were given a SQS grade that did not match that expected based on initial QS, using predefined cutoffs. Main explanations for discrepancies were different perceived $\mathrm{ROI}(40.7 \%)$ and Doppler pixel count near cutoffs between SQS grades $(32.3 \%)$.

Conclusions: We showed that both SQS methods correlated well with QS to assess synovitis, but SQS methods are intrinsically limited when the Doppler pixel count is close to the cutoffs between the SQS grades. Analysis discrepancies between these methods may help further revision of criteria used to assess disease activity with MSUS in RA.

References:

[1] Szkudlarek M et al. Arthritis Rheum 2003.

[2] Hammer HBet al. Ann Rheum Dis 2011.

[3] Qvistgaard E et al. Ann Rheum Dis 2001.
Acknowledgements: We would like to thank all the participating patients, rheumatologists and nurses who made this study possible.

Disclosure of Interest: None declared

DOI: 10.1136/annrheumdis-2017-eular.1149

\section{FRI0627 ULTRASOUND HAND EXAMINATION IS MORE SENSITIVE IN DIAGNOSING HAND OSTEOARTHRITIS THAN CONVENTIONAL RADIOGRAPHY: COMPARISON BETWEEN DIFFERENT ULTRASONOGRAPHIC SCORES}

S. Hussain ${ }^{1,2}$, P. Sivakumaran ${ }^{1,2}$, C. Ciurtin ${ }^{2} .{ }^{1}$ University College London Medical School; ${ }^{2}$ Department of Rheumatology, University College London Hospitals NHS Foundation Trust, London, United Kingdom

Background: Hand osteoarthritis $(\mathrm{OA})$ diagnosis is based on a combination of clinical, imaging features and assessment of risk factors, together with clinical associations and outcomes (1). In a real-life context, clinicians face difficulty in differentiating between $O A$ and other hand arthropathies, particularly when the clinical examination is equivocal (e.g. no obvious bony enlargement with the characteristic distribution for hand $\mathrm{OA}$ ).

Objectives: This is the first study to investigate the usefulness of a standardised ultrasound (US) examination protocol for hand joints in diagnosing hand osteoarthritis (OA) and the correlations between several US scores and clinical, inflammatory and radiographic parameters, aiming to explore which type of investigations are the most useful for diagnosing hand OA.

Methods: We conducted a cross-sectional study including 62 patients, ultimately diagnosed with hand OA based on the ACR diagnosis criteria (2). We compared the 34 joint score of the hand, with smaller, pre-defined joint scores including two scores of 22 and 12 joint each, and another 10 and 6 joint scores for OA. We correlated the US findings with radiographic scores (2108 joints).

Results: Radiographic osteophyte scores correlated very well with the predefined US scores detailed above $(R=0.381$ to $0.645, P<0.05)$, despite having a low sensitivity for detection of osteophytes $(58.6 \%)$, and an even lower sensitivity for detection of erosions (38.4\%) when compared with the 34 joint US score. There was a good correlation between different US scores $(R=0.53$ to $0.97, P<0.05)$, apart from the 6 joint score excluding the proximal interphalangeal joints $(R=$ -0181 to $0.207, P>0.05$ ).

Conclusions: US examination of the hands can facilitate the diagnosis of hand OA in patients who do not fulfil the ACR criteria, by identifying the presence of osteophytes with the particular distribution and number required for diagnosis in a proportion of patients that was three times higher than that of patients diagnosed based on clinical examination and hand radiography alone.

References:

[1] Zhang W, Doherty M, Leeb BF, Alekseeva L, Arden NK, Bijlsma JW, et al. EULAR evidence-based recommendations for the diagnosis of hand osteoarthritis: report of a task force of ESCISIT. Annals of the rheumatic diseases. 2009 Jan;68(1):8-17. PubMed PMID: 18250111. Epub 2008/02/06. eng.

[2] Altman R, Alarcon G, Appelrouth D, Bloch D, Borenstein D, Brandt K, et al. The American College of Rheumatology criteria for the classification and reporting of osteoarthritis of the hand. Arthritis and rheumatism. 1990 Nov;33(11):1601-10. PubMed PMID: 2242058. Epub 1990/11/01. eng.

Acknowledgements: S. Hussain and P. Sivakumaran contributed equally to this study.

Disclosure of Interest: None declared

DOI: 10.1136/annrheumdis-2017-eular.2713

\section{FRI0628 ULTRASOUND SHOWS SIGNS OF INFLAMMATION IN MOST PATIENTS WITH RHEUMATOID ARTHRITIS IN LONGSTANDING CLINICAL REMISSION, IRRESPECTIVE OF CONVENTIONAL SYNTHETIC OR BIOLOGIC DMARD THERAPY}

U. Møller Døhn, C.H. Brahe, M.L. Hetland, V. Fana, S. Krabbe, M. Ammitzbøll-Danielsen, M. Østergaard, L. Terslev. Center for Rheumatology and Spine Diseases, Rigshospitalet, Glostrup, Denmark

Background: None of the currently accepted remission criteria in rheumatoid arthritis (RA) incorporate inflammation on imaging. Signs of inflammation on ultrasound (US) and magnetic resonance imaging are frequently seen in RA patients in clinical remission.(1-3) It is not known whether patients in longstanding clinical and radiographic remission obtained through a DAS28 driven treat to target (T2T) strategy by conventional synthetic disease modifying anti-rheumatic drugs (csDMARD) or by biologic (bDMARD) therapy differ with respect to US detected synovitis.

\section{Abstract FRI0626 - Table 1}

Cut-offs between grades ${ }^{*}$ Sensitivity and specificity in the different statistical models

\begin{tabular}{|c|c|c|c|c|c|c|c|c|}
\hline & \multirow{2}{*}{\multicolumn{2}{|c|}{ Raw data }} & \multirow{2}{*}{\multicolumn{2}{|c|}{ Model 1}} & \multirow{2}{*}{\multicolumn{2}{|c|}{ Model 2}} & \multirow{2}{*}{\multicolumn{2}{|c|}{ Model 3}} \\
\hline & & & & & & & & \\
\hline & Sensitivity & Specificity & Sensitivity & Specificity & Sensitivity & Specificity & Sensitivity & Specificity \\
\hline Grade $0-1$ & 1.0 & 1.0 & 1.0 & 1.0 & 1.0 & 1.0 & 1.0 & 1.0 \\
\hline Grade $1-2$ & 0.94 & 0.72 & 0.99 & 0.85 & 0.99 & 0.82 & 0.96 & 0.77 \\
\hline Grade $2-3$ & 0.90 & 0.84 & 0.99 & 0.92 & 0.99 & 0.91 & 0.98 & 0.92 \\
\hline
\end{tabular}

${ }^{*}$ Cut-off between grade 0 (G0) and G1: 0\%; between G1 and G2: $10 \%$; between G2 and G3: $50 \%$. 